\title{
LONG-LIVED PLASMA PROCESS, CREATED BY IMPULSE DISCHARGE IN MICRO-DISPERSE DROPLET ENVIRONMENT
}

\author{
Serge Olszewski*, Tamara Lisitchenko, VitaliJ Yukhymenko \\ Taras Shevchenko National University of Kyiv, Radio Physics Faculty, Pr. A. Glushkova 4G, Kyiv, Ukraine \\ * corresponding author: olszewski.serge@gmail.com
}

\begin{abstract}
The processes of organic compound (phenol and cation-active surfactants) destruction in water solutions, which stay under the influence of plasma treatment have been investigated in different dynamic plasma-liquid systems (PLS) with discharges in droplet micro-disperse environments (DMDE). The long-lived plasma process with separate spectral properties has been observed for pulsed discharge in DMDE. The approximate computer model is being proposed for a description of this effect. According to the introduced model this long-lived process is the aggregation of correlated discharges between charged droplets.
\end{abstract}

KEYWORDS: dynamic plasma-liquid system, plasma-chemical processing, ultrasonic nebulization, droplet micro-disperse environment.

\section{INTRODUCTION}

Water is a valuable natural resource. With metabolic processes in all aspects, forming the base of human living, water plays an exclusive role. Methods based on plasma-chemical processes in the liquid-gas environments for water treatment and purification of highly polluted wastewater are the most promising among the others. Unlike the regenerative methods which remove the collected impurities from the water into the solid (absorption), gas (desorption) or nonaqueous liquid (extraction) phase, the destructive method (technology of water and industrial waste plasma-chemical processing) is based on conversion of the chemical structure of molecules and impurities.

The problem of complete cleaning of the industrial wastewaters from organic highly active and toxic substances (HATS) is important enough and simultaneously difficult to apply. However this problem can not be solved completely. Apparently, plasmachemical technologies are represented by the most perspective ones, as allowing achieving quick speed of substances destruction under the conditions of expense of high-energy concentration. However, it is necessary to take into account that chemical reactions occurring in the liquid phase can be stimulated only by particles that penetrate from plasma into the liquid. They can be active radicals created as a result of recombination processes in the plasma phase. They could also be excited molecules that are generated as a result of the bombardment of liquid surface by charged particles of plasma. Therefore the effectiveness of plasma-liquid systems (PLS) as applications for liquid treatment is primarily determined by the size of the interaction zone between liquid phase and plasma.

The integral feature for all systems with plasmaliquid interaction is their little active volume as com- pared with total liquid volume. As a matter of fact, the active volume of plasma-liquid systems is defined as the contact area multiplied by the thickness of the diffusion layer of active plasma particle in liquid. The contact area is the area of surface of contact plasma and liquid.

One of the directions to increase the ratio of active volume to total liquid volume is the using of PLS with liquid solutions in micro-disperse state. For example, PLS are based on discharges in fog that contains drops with size of order diffusion layer thickness. Using of the pulsed discharges as sources of decaying plasma in droplet environment can generate the flows of chemical-active particles onto extended surface of liquid. At the same time, the small size of discrete droplets provides more complete treatment of the total liquid volume.

Some features of plasma-liquid systems based on pulsed discharges in the droplets micro-disperse environments have been studied in the present work.

\section{MATERIAL AND METHODS}

The scheme and photo of experimental setup are represented in Fig. 1. In this setup the distilled water (4) was sprayed by ultrasound field into inside volume of working vessel (3) and transformesd to monodisperse fog (10).

The quartz cylinder with inside diameter $28 \mathrm{~mm}$ and height $150 \mathrm{~mm}$ was used as a working vessel. The ultrasound field was created by quartz crystal (8). The friction of an ultrasound field was $800 \mathrm{~Hz}$ and acoustic power $\sim 60 \mathrm{~W}$. For initiating a spark discharge between copper electrodes (1), high voltage $\sim 10 \mathrm{kV}$ was input onto them. The discharge gap between electrodes was $3 \mathrm{~mm}$. The gap between high-voltage electrode and inside surface of quartz vessel was $5 \mathrm{~mm}$. The current of spark was measured by Rogowski coil. 


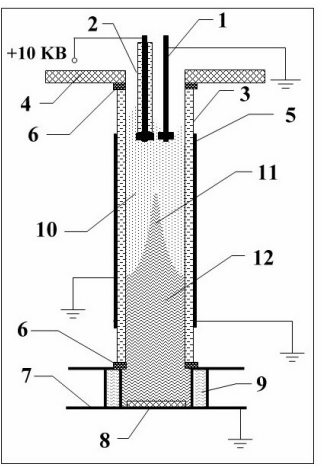

a)

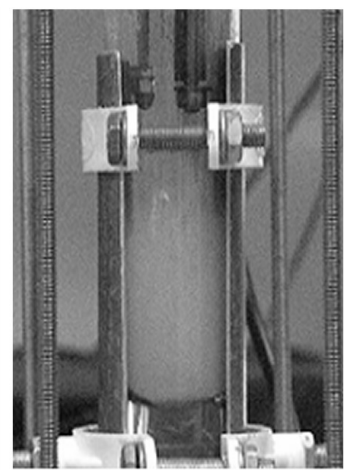

b)
Figure 1. Experimental setup: a) scheme, b) photo; 1 - metal electrodes, 2 - quartz insulator, 3 - quartz vessel, 4 - up flanges, 5 - side metal walls, 6 - rubber seals, 7 - ultrasonic sprayer, 8 - quartz piezocrystal, 9 - water cooler, 10 - ultrasonic fog, 11 - ultrasonic fountain, 12 - work liquid.

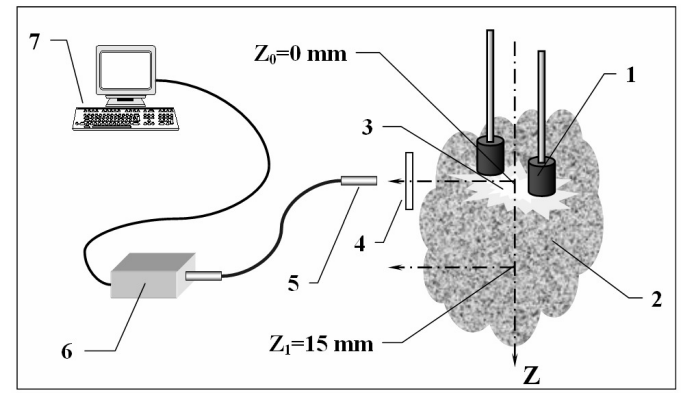

Figure 2. Diagram of optical measurements; 1 - copper electrodes, 2 - droplet environment, 3 - pulsed discharge, 4 - filter wheels, 5 - optical-fiber waveguide, 6 - CCD-spectrometer, 7 - PC-recorder.

The value of current was $\sim 1 \mathrm{kA}$. All processes were observed in atmospheric pressure.

The emission spectra were registered by CCDspectrometer SOLAR TII. The scheme of optical measurements is shown in Fig. 2 .

The coordinate axis $z$ was directed along the geometrical axis of system down. The point $z=0$ was located in the horizontal plane contained discharge gap. The optical radiation was collected along axis directed perpendicularly to axis $z$ through the filter wheel (4) into optical-fiber lightwaveguide (5). The spectra registered by CCD-spectrometer (6) were stored by specialized software in personal computer (7).

The typical lead time for slow processes in the volume of system was investigated by analysis of images from video-frames. Video filming was performed by video camera EAN850A with duration of videoframe $30 \mathrm{~ms}$. The video-frame duration itself determined the interval between time readings in experiment. According to the result of test-drives for different video-cameras [7, 9, 8, the typical time interval of CCD-matrix perception is $\sim 1 \mu \mathrm{s}$. This time interval determined the experiment accuracy in time for slow processes.

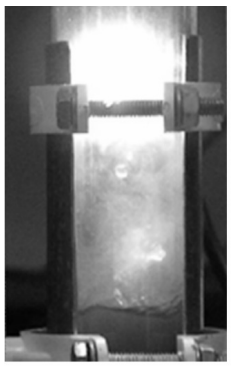

a)

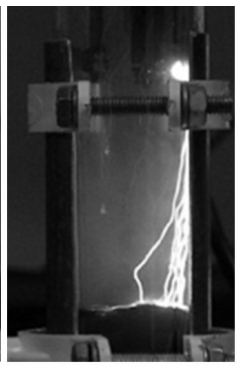

b)

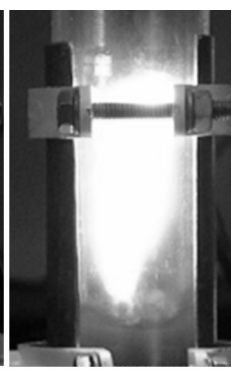

c)
Figure 3. Photo of plasma processes in micro-disperse droplet environment, a) spark discharge between metal electrodes, b) sliding discharge along inside surface working vessel between high-voltage electrode and water surface, c) long-lived plasma process with spectral properties different from the previous discharges types.

\section{Results AND Discussion}

\subsection{EXPERIMENT}

In the course of experiment three different processes in working volume of system were being observed (Fig. 3).

Fig. 3 a shows the trivial spark discharge between metal electrodes. The breakdown voltage of this discharge in water fog was $\sim 7 \div 10 \mathrm{kV}$. The pulsing current had amplitude $\sim 1.5 \mathrm{kA}$. This discharge had a single channel. The visual lengthwise size of spark channel was $\sim 10 \mathrm{~mm}$. The visual diameter of spark channel was $\sim 1 \mathrm{~mm}$. The duration current impulse of spark discharge was $\leq 10 \mu$ s.

Fig. 3p is shows the sliding discharge between high-voltage metal electrode and liquid surface. The breakdown voltage of this discharge was $\sim 10 \mathrm{kV}$. The pulsing current of sliding discharge had amplitude $\sim 1.5 \mathrm{kA}$. This discharge had a multiple channels located near inside dielectric surface of working vessel. The visual lengthwise size of these channels was $\sim 50 \mathrm{~mm}$. The visual diameter of channels was $\sim 1 \mathrm{~mm}$. The duration of current impulse of sliding discharge was $\sim 10 \mu \mathrm{s}$.

Fig. 3r shows the volume process with glow color distinct from the spark and gliding discharges. On the video the evolution of this process was placed on several consecutive video-frames. As a rule the number of these video-frame was $\geq 4$. In some cases their number attained 18. The typical live time of this process was determined by calculation of these video-frame number. This live time was $\sim 120 \div 540 \mathrm{~ms}$. This process did not have channels and had a look of transparent luminous cloud. As systematical observations have shown, the necessary condition for start-up of longlived process is fog existence in area of this process.

For the cases of impulsing discharges the breakdown voltage was measured by high-voltage voltmeter, built in power source for reservoir capacitor. The reservoir capacitor was used for energy supply into discharge and in times of discharge breakdown power source was detached from this capacitor automatically. However 
this on-board voltmeter did not register the voltage in system during the time of volume process existence. The discharge current was not registered either. The maximum output current of power source was $150 \mathrm{~mA}$. Therefore during the time of volume process existence the current in system was $\leq 150 \mathrm{~mA}$.

The results of spectroscopic investigations of impulsing discharges in PLS with droplet micro-disperse environment are shown on Fig. 4. The emission spectrum of spark discharge is represented on plot a) and spectrum of sliding discharge on b). The spark spectrum was registered along axis with coordinate $z=0 \mathrm{~mm}$. The radiation of sliding discharge was registered along axis with coordinate $z=15 \mathrm{~mm}$ (Fig. 2). This axis was distanced from place of spark purposely to avoid the overlapping of spectra of different discharges. Also this method was used for registration of spectra of longlived process too. The atomic lines of hydrogen was $\lambda=657.8 \mathrm{~nm}$, of oxygen $\lambda=778.8 \mathrm{~nm}$ and material of electrodes $\lambda=229.2 ; 327.8 ; 524.8 \mathrm{~nm}$ are present in spectra for both cases. In these experiments it was the atomic lines of copper. The molecular bands of hydroxyl $(286.3 \div 343.7 \mathrm{~nm})$ were not registered in radiation of impulsing discharges.

The probably cause of absence of hydroxyl bands in emission spectra of both pulsed discharges can be related with presence of metal vapor. The metals have the most low excitation potentials of electron states. Therefore the high concentration of metal atoms can to increase appreciably probability of radiationless deexcitation of hydroxyl molecules. But this supposition requires of screening and goes beyond the scope of this work.

The emission spectrum of long-lived process is represented on Fig. 5

Optical radiation of long lived process was registered along axis with coordinate $z=15 \mathrm{~mm}$. The atomic lines of hydrogen and oxygen were also present in this spectrum. However unlike cases of impulsing discharges the power band of hydroxyl was present and atomic lines of electrodes material were absent in emission spectrum of long-lived process. The absence of atomic lines of copper in emission spectra of longlived process can to explain by hypothetical nature of this process. The long-lived process can be related with discharge between charged drops. If it is true then the material of electrodes in this case is the water and atomic lines of copper can not be present in emission spectra.

The spreading of long-lived process in working volume is shown by serial video-frames on Fig. 6

As a result of processing of images from these videoframes the estimation of linear speed of glow area spreading was made. Estimated rate of glow area boarder spreading was $\sim 0.7 \mathrm{~m} \mathrm{~s}^{-1}$. Such a small value of this speed specifies that the mentioned process cannot be propagation of a combustion wave.
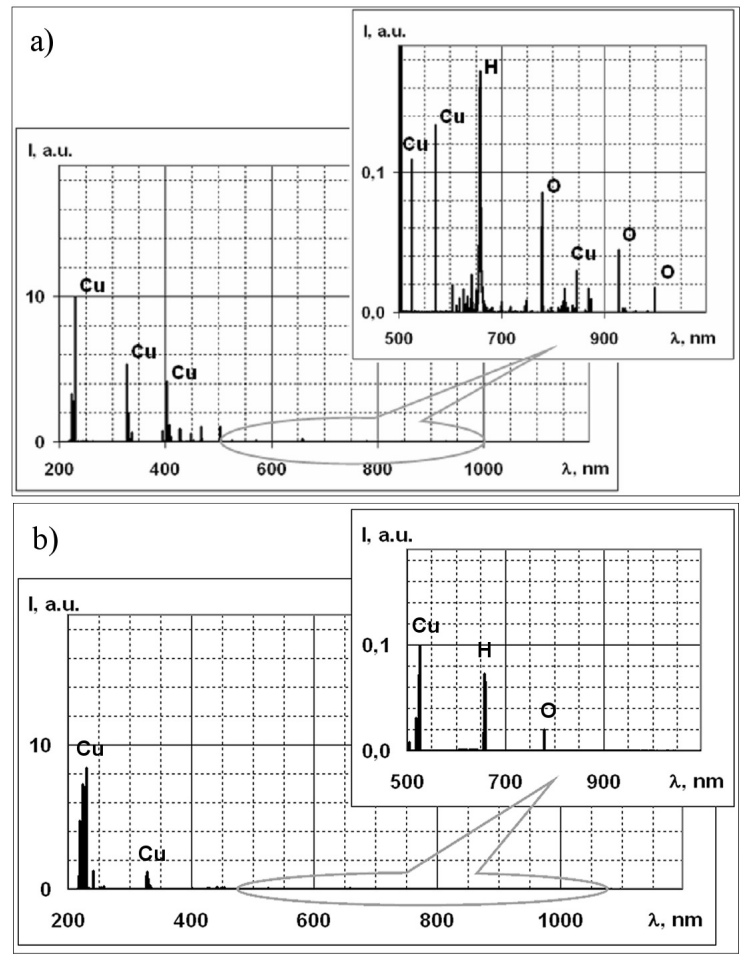

FIGURE 4. Emission spectra of impulsing discharges in PLS with micro-disperse droplet environment, a) spark discharge, $z=0 \mathrm{~mm}$, b) gliding discharge $z=$ $15 \mathrm{~mm}$.

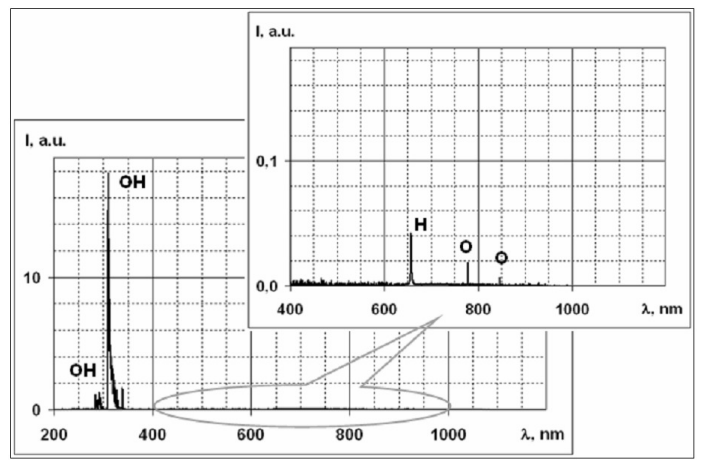

Figure 5. Emission spectra of long-lived processes in PLS with micro-disperse droplet environment $z=$ $15 \mathrm{~mm}$.

\subsection{PhYSICAL MODEL}

Statistical analysis of experimental data has shown that the necessary conditions of durable process appearing are obligatory presence of water fog in the volume with discharge. The hypothesis, that long-lived process for given conditions is the correlated multiple spark (CMS) discharge between charged and uncharged fog drops during their approaching has been introduced. To verify this hypothesis, the approximate model of long-lived process was created. According to the model, fog drops located in the area impulsing discharge channel gain an electric charge due to their contact with plasma. In the result of the Brownian motion they are mixed in the volume with the uncharged fog drops. Chaotic motion of aerosol particles 


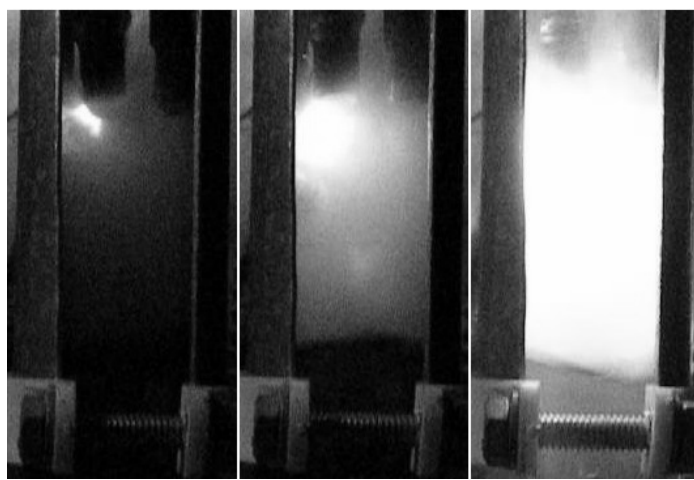

FiguRE 6. Spreading of long-lived process in volume on serial video-frames.

leads to the approaching of single drops on the lengths in order of magnitude of their radii. In the case of such approaching between the fog particles with different charges, or between charged and uncharged particles, the electric field appears with the magnitude that can be much larger (according to [5, 3]) then the one calculated using the Coulomb's law. Electric fields' value between the single particles is also related to self-consistent electric field, formed by the ensemble of charged drop, chaotically distributed in the working space. Spatial redistribution of charged particles in time is defined by the Langevin equation with the additional determinate force, which has an electrostatic nature,

$$
\xi_{\operatorname{tr}}^{x} \frac{\mathrm{d} x}{\mathrm{~d} t}=f_{x}(t)+F_{x},
$$

where $\xi_{\operatorname{tr}}^{x}$ is drag coefficient for a ellipsoidal drop, $f_{x}(t)$ is random force and $F_{x}$ is external electrostatic force.

According to [5], the drop can be split into the parts as a result of Rayleigh or Taylor instability. As far as Taylor instability depends on the outer electric field and can be developed even for the electro neutral drops, in case of charged aerosol drops ensemble it is more probable. According to [6], charged liquid drop always acquires an ellipsoidal form. An instability criterion for ellipsoid drops is given by the inequality

$$
\frac{\varepsilon_{0} E_{\mathrm{S}}^{2} R}{4 \alpha_{\mathrm{S}}} \leq 1.54
$$

according to Taylor 4, wherein $R$ is the drops' radius, $\alpha_{\mathrm{S}}$ is coefficient of surface tension, $E_{\mathrm{S}}$ is the value of outer electric field and $\varepsilon_{0}$ is absolute dielectric permittivity. The conditions of spark ignition discharge between the drops, according to [2], are defined by the electric field value in $\mathrm{kV} / \mathrm{cm}$ as

$$
E_{\mathrm{S}}=27.2\left(1+\frac{0.54}{\sqrt{R}}\right),
$$

wherein $R$ is radius of drops.

The conditions of spark breakdown require more intensity of the electric field, comparing to the case of drops' break-up according to the capillary surface instability of Taylors' criterion. In cases of quasistatic systems, spark breakdown between micro-drops of electro conductive liquid is low probable. But according to [1] the typical time of capillary instability development can be estimated as

$$
\tau \sim R^{2} \rho^{1 / 2} \alpha_{\mathrm{S}}^{1 / 2}
$$

wherein $\rho$ is liquids' density. This time range is more than in 6 times of magnitude greater than the time range of spark discharge. Hence, in dynamic systems the conditions can be carried out when the electric field between neighbor drops can increase to the value enough for spark discharge ignition during period of time less than the typical time of instability development. Such an increasing of the electric field can be provided either by rate of charged and uncharged drops approaching, or by the superposition of charged aerosol drops' self-consistent field and vortex electric field produced by the alternating current of spark discharge between neighbor pair of drops. The latter mechanism is also an extra discharges correlation factor between the approaching drops' pairs, because it relieves the breakdown conditions due to the photoelectric effect and charged particles diffusion, and leads to the impulse increasing of the electric field value.

\subsection{Simulation}

According to presented physical model, 3D computer model has also been developed. Due to the orbital symmetry of ellipsoidal drops, the number of dimensions can be reduced to $2 \mathrm{D}$, so the working space was chosen more like the experiment only for two coordinates, and the third dimension was chosen as contracted in two orders of magnitude: $0.02 \times 2 \times 10 \mathrm{~cm}$. Fogs density was chosen as $5 \times 10^{3} \mathrm{~cm}^{-3}$.

On the first step of calculation the ensemble of fog drops was created, with Gamma-distributed characteristic sizes and random coordinates inside the working space,

$$
n(a)=\frac{\mu^{\mu+1}}{\Gamma(\mu+1)} \frac{a^{\mu}}{r_{\mathrm{m}}^{\mu+1}} \exp \left(-\mu \frac{a}{r_{\mathrm{m}}}\right),
$$

wherein $\Gamma(\mu+1)$ is gamma function, $r_{\mathrm{m}}$ is the most probable drops radius and $\mu$ is half-width of distribution.

Initial velocities of particles were generated according to the Maxwell distribution. For each $N$-th particle random charge was specified, so that value $1 / N$ could be distributed in space according to the Gaussian law.

On the next step self-consistent electric field in instant coordinate of each particle was calculated.

Then, for each pair of particles the correction to the electric fields' intensity [3, 6], criterion of electrical break-down - Eq. 3, and particles' break-up criterion were calculated - Eq. 2. For the pairs of drops, which conformed to the breakdown criterion, the break-down impulse discharge current was calculated. For the drops conform to the break-up criteria, 
the initial time point of instability development was fixated. If in the process of further evolution integral time, during which the break-up criteria was fulfilled, exceeded the time of instability development, such pair of drops was replaced by the ensemble of drops with typical sizes and integral charge according to 4 . For the particles conform to the criterion of corona discharge

$$
\left\{\begin{array}{l}
\tau_{\text {approach }} \leq R^{2} \rho^{1 / 2} \alpha_{\mathrm{S}}^{1 / 2} \\
E_{\mathrm{S}} \geq 50 \mathrm{kV} \\
r \geq 10 R
\end{array}\right.
$$

the loss of charge took place, been calculated as the discharge current multiplied by the time step. The pair of drops, approaching at the distance equal to the sum of their radii, was replaced by the one particle with the integral volume and charge. On the next step, the Langevin equation Eq. 1 was numerically solved for each drop, and on the next step the recalculation of particles ensemble spatial evolution was took place. After that, iteration was repeated. Calculations stopped when the linear velocity of glow boundary of CMS-discharges area was formed.

As simulation result it was calculated how the areas that contained multiple spark discharges between drops were propagated in space. The evolution of simulated CMS-discharge is shown in Fig. 7.

This evolution is represented by space distribution of spark current in different time stations. The black points correspond to CMS-discharge state after $25 \mathrm{~ms}$ of charged cloud creation. The dark grey points correspond to time station $50 \mathrm{~ms}$ and light-grey points to $100 \mathrm{~ms}$.

The comparison of experiment and simulation results is presented in Fig. 8 .

This plot represents the dependence of border location of long-lived process glow area upon time. The black curve with round points corresponds to results of experimental measurement. The grey curve with triangular points corresponds to the results of simulation. The experimental estimated average value of linear speed of long-lived process spreading is $0.7 \mathrm{~m} \mathrm{~s}^{-1}$. The calculated rate of CMS-discharge area boundary spreading is $\sim 0.4 \mathrm{~m} \mathrm{~s}^{-1}$. This result is conformed to experimental measured values.

The mean radius of drops that support CMSdischarge is $\sim 0.8 \div 1 \mu \mathrm{m}$. For drops' size $\leq 10 \mu \mathrm{m}$ the atomization probability is greater then probability of spark breakdown, because for this case drops have smaller mean velocity and capillary instability has enough time to progress. For drops' size $\leq 0.5 \mu \mathrm{m}$ the probability of lost charge in the result of corona discharge is greater then probabilities of the other considered remainder elementary processes of the drops state transform.

The increase of self-consistent field value reduces the probability of spark breakdown, since it relieves the conditions of capillary instability development. However the field gradient enhances condi-

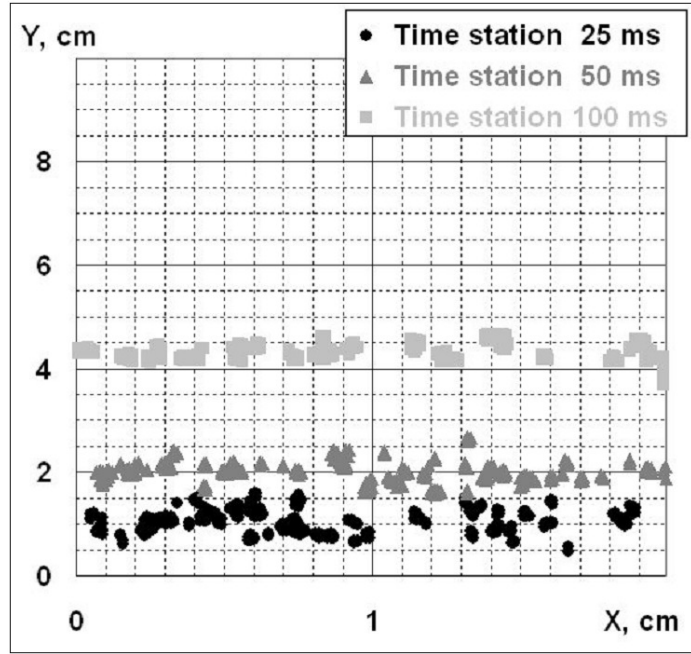

FiguRE 7. Space distribution of areas that contained pairs of drops with spark discharges between it.

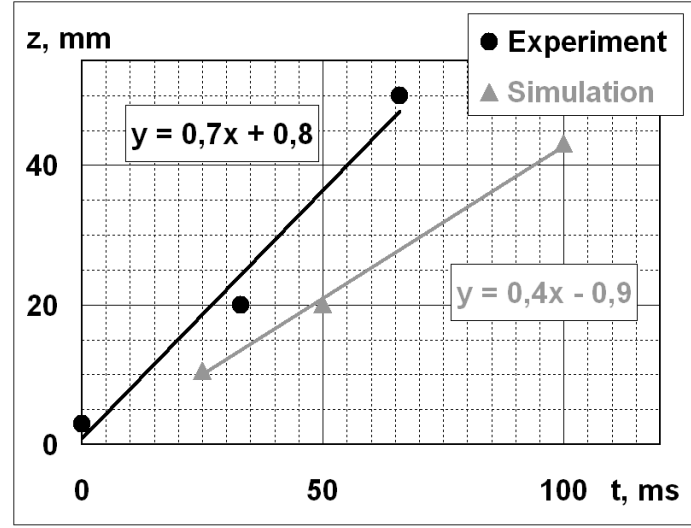

Figure 8. Dependence of position of glow border on time; the black curve represent results of experimental measurements; the grey curve represent results of simulation.

tions of spark breakdown for sufficiently rapid drops. On the periphery of charged fog cloud the gradient of self-consistent field mainly hinders the electrical field increase during drops approach. It is right for charged drops that are moved from charged cloud to periphery. But chaotic pattern of drops movement provides a grate drops number with velocity that is oriented to self-consistent field. Just the ensemble of these drops is the main source of CMS-discharge existence.

\section{Conclusions}

Comparison of calculation and experimental results gives the following conclusions:

- In terms of the introduced model, long-lived plasma process created by the impulsing discharge in micro-disperse droplet environment can be presented as correlated multiple spark discharge (CMSdischarge) between discrete pairs of differentlycharged drops. 
- The self-consistent electrical field of charged droplets ensemble decreasei the probability of CMSdischarge initiation on periphery of charged fog cloud and increase the probability of droplet fragmentations due to Taylor instability.

- The calculated speed and experimentally measured speed of propagation of glow boundary of CMSdischarge match together within the order of magnitude. This speed corresponds to submicron size of drops that predominately support the CMSdischarge.

\section{ACKNOWLEDGEMENTS}

The present work was partly supported by the Kyiv National Taras Shevchenko University, by the National Academy of Sciences of Ukraine, by the Ministry of Education and Science of Ukraine.

\section{REFERENCES}

[1] D. F. Belonoszko, A. I. Grigoriev. The characteristic time of instability droplets charged to rayleigh limit. Technical Physics Letters 25(15):41-45, 1999.
[2] E. D. Lozanskij, O. B. Firsov. The theory of the spark. Atomizdat, Moscow, 1975.

[3] M. V. Mirolubov, et al. Methods for calculating electrostatic fields. Higher School, Moscow, 1963.

[4] V. M. Muchnik, B. E. Fishman. The electrification of coarse aerosols in the atmosphere. Gidrometeoizdat, Moscow, 1982.

[5] V. A. Saranin. Some of the effects of electrostatic interaction of water drops in the atmosphere. Technical Physics 65(12):12-17, 1999.

[6] A. A. Shutov. The form of drops in a constant electric field. Technical Physics 72(12):15-22, 2002.

[7] A. Zajtsev, et al. Testing of a network television camera Samsung SNP-5200H. PROSystem CCTV 1:28-45, 2012

[8] A. Zajtsev, et al. Testing of a network television camera Sony SNOEM52. PROSystem CCTV 2:60-72, 2012 .

[9] A. Zajtsev, et al. Testing of the network video registrar Softtera NVR-SR5Q24. PROSystem CCTV 1:46-53, 2012. 